\title{
Bridge Safety Monitoring Technology Based on Risk Traceability Mechanism
}

\author{
Yong-Hui ZHONG ${ }^{1, a *}$, Yun-Biao DONG ${ }^{2, b}$, Lei WANG ${ }^{3, c}$ \\ ${ }^{1}$ Chongqing Jiaotong University, Xuefu Avenue No.66, Nan'an District, Chongqing City, China \\ ${ }^{2}$ China Water Conservancy and Hydropower Engineering Bureau seventh Engineering Bureau Co., \\ Ltd. \\ ${ }^{3}$ CCCC Second Harbor Engineering Co. Ltd \\ atopjmu15@sina.com, b1767734318@qq.com, '1347814846@qq.com
}

${ }^{*}$ Corresponding author

Keywords: Deflection Monitoring, Video Monitoring, Risk Traceability.

\begin{abstract}
As the bridge structure is effected by the environment, the erosion of harmful substances, vehicles, wind, earthquakes, fatigue, and other human factors, plus the degradation of the material properties, parts of the structure suffer varying degrees of injury before reaching their design life. This paper constructed deflection video surveillance systems through the analysis, including video surveillance system consisting of hardware, design features and recognition module, as well as traceability of risk monitoring database design, wireless remote real-time bridge health monitoring.
\end{abstract}

\section{Introduction}

Many parts of the structure will suffer varying degrees of injury before reaching their design life as the bridge structure is effected by the environment, the erosion of harmful substances, vehicles, wind, earthquakes, fatigue, and other human factors as well as the degradation of the material properties in the construction and use of the bridge[1]. If these damage can't be inspected and maintained in time, it will lightly cause road safety problems and short the life of the bridge, or it will heavily lead to the sudden destruction and collapse of the bridge[2].

In order to monitor the safety of the bridge structure, a monitoring system included monitoring contents such as deflection, inclination, acceleration and stress is constructed to realize wireless, real-time and long-distance bridge health monitoring. The hardware part of the monitoring system is consist of a series of sensors that monitor data in a proactive way and transmit the data back to the backend database. Although the monitoring system can continuously monitor the structural safety status of the bridge from all aspects, it only judges the moment and states of the structure risk and provides no information about the cause of the risk, which is unfavourable to find out the cause of the security risk and analyse the bridge structure performance evolution. In fact, the structure risk of the bridge is often caused by the intense down-warping of the beam that caused by overweight vehicle driving, in other words, when the bridge appears a greater deflection, we need to get information of vehicles that drive on the bridge at the same time, especially the suspicious overweight vehicles' license plates as the important supplementary information for the bridge health monitoring, that is why we put forward the question of traceability of bridge safety risk.

\section{The Risk Traceability Technology System Based on the Abnormal Deflection Trigger}

The largest physical quantity associated with a safety risk is the deflection. The deflection is actively collected by the deflection sensor. Therefore, there are two steps we need to complete to obtain the vehicle information at the down-warping moment. Firstly, sync continuous collected videos' information, save photos of vehicles and recognize vehicle license plates[6]. Secondly, connect the video information and the deflection information in the database, then screen out suspicious vehicles. The technical path is shown in Figure 2.1. 


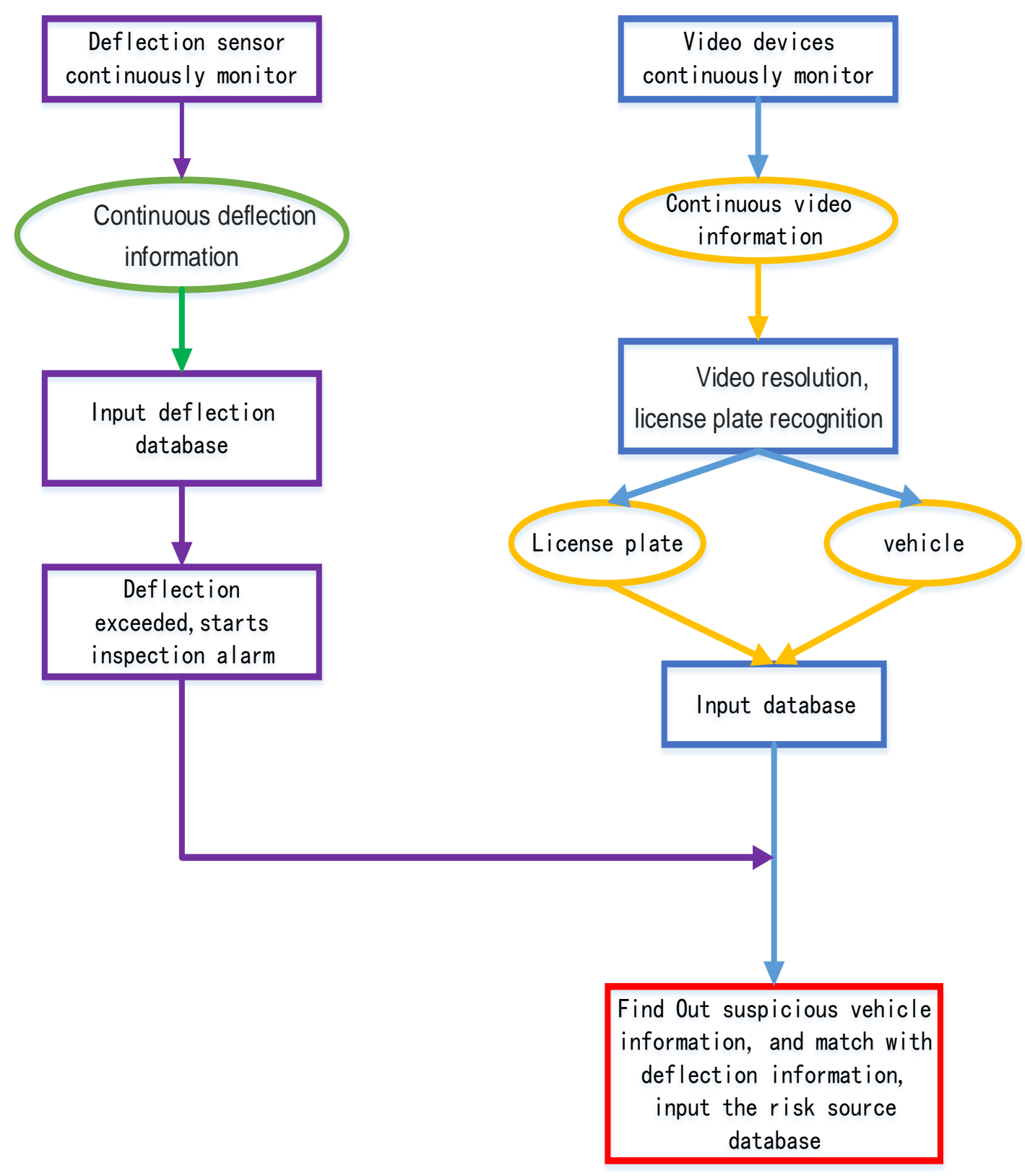

Fig. 2.1 Risk traceability technology path graph

\section{Bridge Safety Monitoring System Design Based on the Risk Traceability Mechanism}

\section{The Construction of Video Surveillance System}

The main purpose of video surveillance system is to film vehicles continually, identify photos, $\mathrm{s}$ ave image information and collect first-hand information on risk traceability.

\section{Hardware Design of Video Surveillance System}

The equipment has the function of detecting the vehicle, capturing the strong light supplement, and recognizing the license plate. The image acquisition module adopts the CCD image sensor. The license plate recognition mode adopts the international advanced computer vision recognition technology which can achieve real-time and high-speed recognition of vehicles in the continuous video and captured images and capture strong supplement light.

The main processing module includes a front-end image acquisition module, a data processing module (dual CPU), a storage module[7]. The structural frame diagram is shown in Figure 3.1 


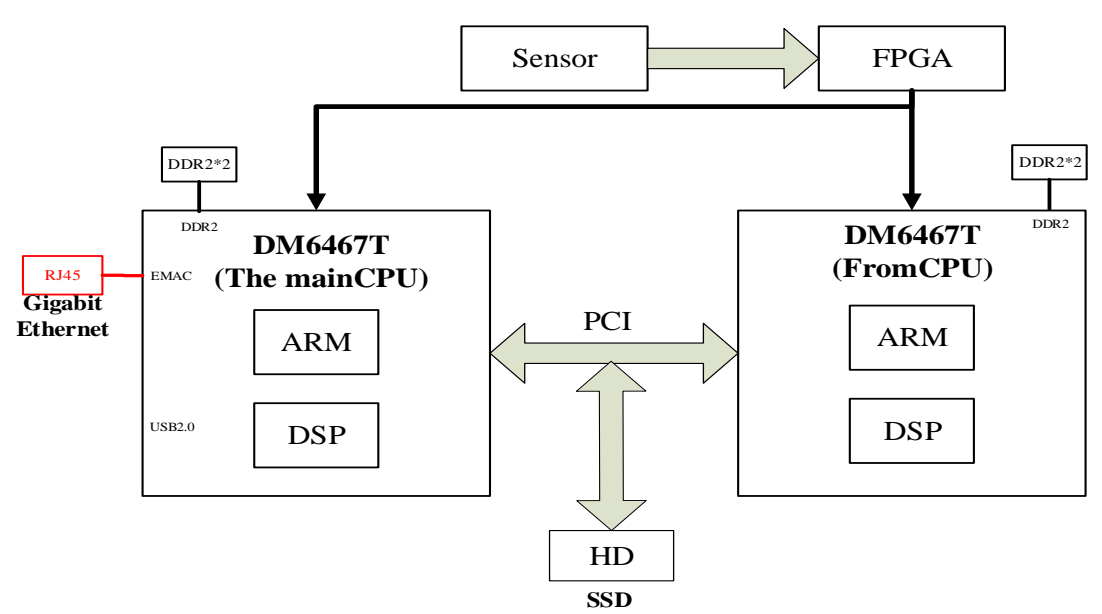

Fig. 3.1 HD video internal structure

The software system is divided into four modules which are image acquisition, tracking and detection of moving objects, license plate detection and recognition, image coding output. The general workflow is shown in Fig. 3.2

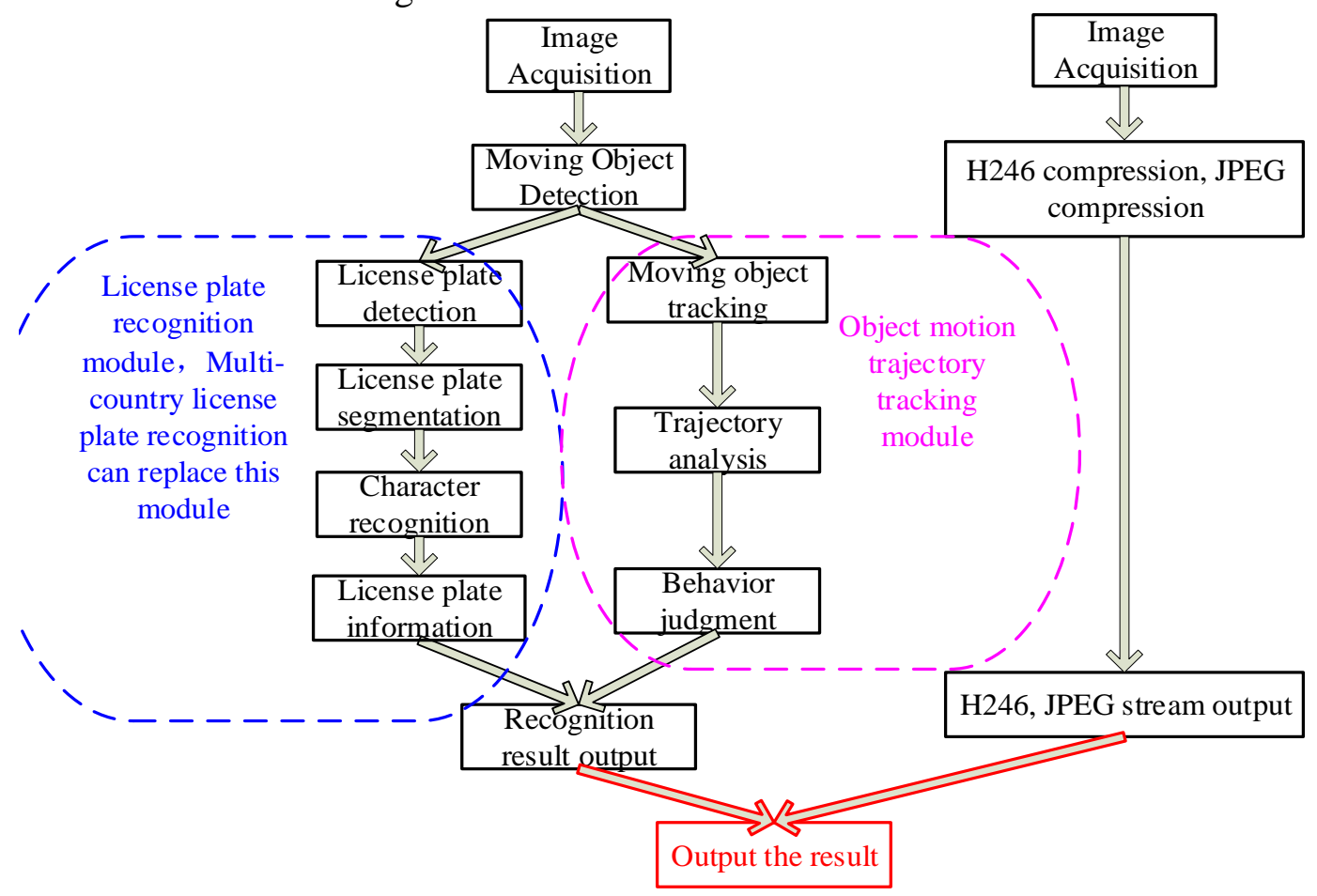

Fig. 3.2 Bayonet HD video machine workflow

\section{Detection and Recognition Module Function}

(1) Vehicle Detection

The device adopts the pure video detection mode, which can automatically detect and recognize the video stream frame by frame in real time without the assistance of ground sense coil.

(2) License Plate Recognition

The device has the license plate recognition function. License plate identification provides the information including license plate images (license plate detection images and license plate binary images), large vehicle images, license plate identification and the color of license plate[7].

(3) Face Recognition Capture

The device can capture all the detected vehicles. And the system can fill the light with a special snap light. The captured image can clearly identify the facial features of the driver (in the front seat), 
taking into account the expression of the license plate without affecting the license plates of the normal recognition.

(4) Body Color Recognition

The device has the body color recognition function which can identify ten body colors according to the standard of GA24.8-2005.

(5) Vehicle Type Judgment

Equipment can determine the type of vehicle, and the vehicle specific dimensions (m) length, width information.

(6) Video Tacho

The device detects, locks and traces moving vehicles in the video stream image, depicts its movement trajectory, measures its displacement, and obtains accurate vehicle velocity.

(7) Press or Cross Line Detection of Cars

The device detects, locks and tracks the moving vehicles in the video stream image, and judges whether the vehicle is pressed or crossed the line according to the vehicle movement path and the user-defined lane separation line and the double yellow line.

(8) Retrograde Violation Detection

The device detects, locks and tracks the moving objects in the video stream image, and judges that the running direction of the vehicle is positive or retrograde according to the trajectory of the vehicle. If the device detects the vehicle retrograde, it will judge the vehicle as illegal and captured the image as proofs.

(9) Picture Overlay Function

The device supports the character overlay function. After the configuration parameters, the device can automatically add the character information to the picture stream .

(10) Adaptive Image Acquisition Parameter Control

The device can automatically determine the brightness of the environment, adjust parameters.

(11) Information Filtering

The device provides information filtering function of license plate recognition. Users can define the type of the recognition results output according to their demands .

(12) PC connection

The device provides three types of connection: identification results data connection, command connection and video streaming connection.

\section{Risk Traceability Database and Back-End Software}

\section{Risk Traceability Database Design}

According to the deflection of the bridge layout sensors, the system can trigger synchronous capture when the number of overloaded vehicles exceeds a threshold. The image capture adopts two modes, active synchronization mode and passive synchronization mode. The system will design a related database, saving the risk traceability data that derived from the match after coordinated deflection[3].

\section{Software Function Design and Development[4]}

(1) Tendency Analysis

Through the long-term accumulation of risk traceability data, the software summarizes the long-term law between the change of bridge deflection and heavy vehicle driving. Taking time as abscissa and risk parameter as ordinate, we will get a trend graph about the parameter changing with time. Through that trend graph, we can know the change of bridge structure condition. It is helpful for predicting the possibility of structural risk in a certain extent.

The method of trend analysis is only based on a long time and a large number of data. Through mathematical statistics, data fitting, and other methods, we can understand the change of structure condition and make an estimate of the healthy development of the structure [5].

If above parameters have changed obviously, and the change is a little large comparing with

theoretical values and experience values, we need to analysis it in detail to find the problem's cr 
ux. On the basis of a large number of data, we also can extrapolate the possible development status of the structure to the preparation of maintenance work ahead.

(2) Risk tracking

In the development of the bridge structure's long-term evolution, we can analyze the fast deteriorating stage and find all the vehicles that are responsible for causing a violent deflection of the bridge and diminution of its fatigue life, it is a powerful explanation of a bridge performance's long-term evolution. In addition, the traceability data information is an extremely valuable first-hand information in some special conditions when there happens major accidents.

\section{Software Development and Application}

The software adopts full-page form development and embedded in the backstage of the whole bridge monitoring system. The home page (Fig. 3.3) shows the latest data values, including temperature and humidity, traffic flow, wind information, etc.

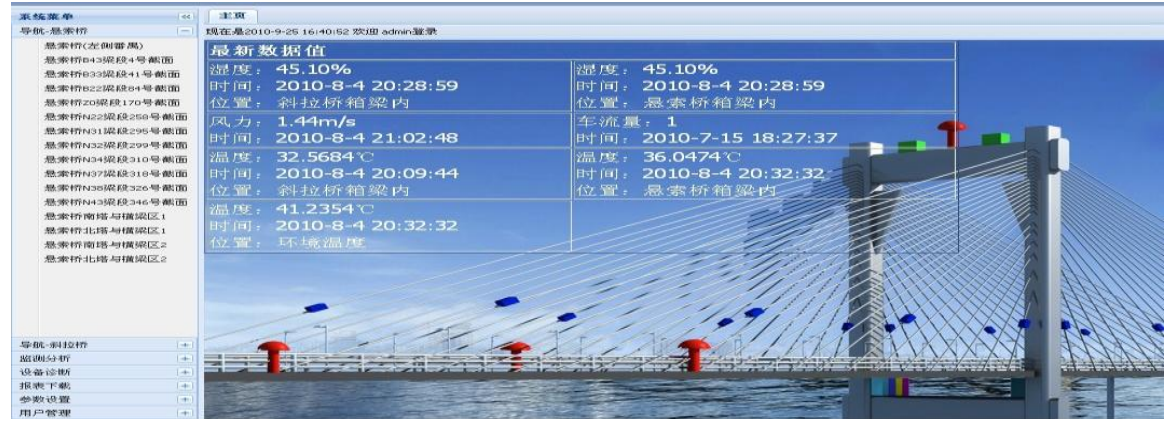

Fig. 3.3 Software Home Page

The main interface of the system will appear three icons in the upper right corner, click the icon to view the warning information, modify the password, exit, as shown in Figure 3.4

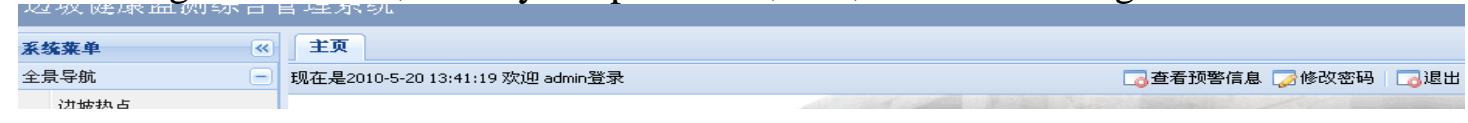

Fig. 3.4 System management function keys

Click to view the warning information icon, interface will be pop-up (Figure 3.5), you can query the corresponding deflection value, time, video information, license plate number, etc.

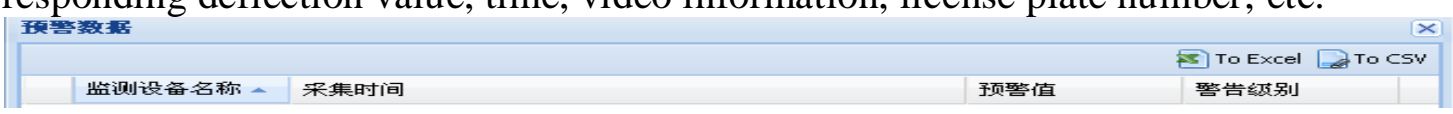

Fig. 3.5 View the warning information

Client can click on the icon in the upper right corner to save data. Shown in the figure 3.6

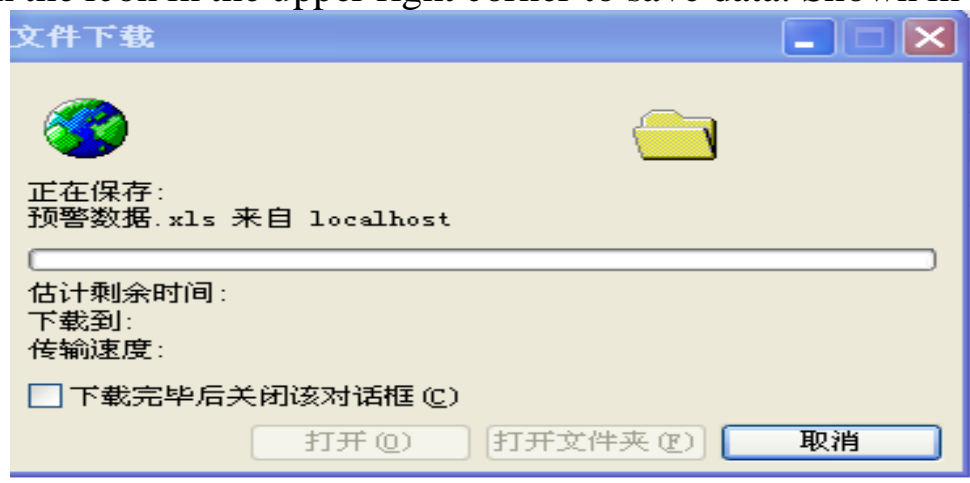

Fig. 3.6 Save the data to local 


\section{Conclusion}

In this paper, we constructed the deflection video surveillance system that includes the hardware composition, function and recognition module design, and the risk traceability surveillance database and backstage software design, which achieved the wireless real-time bridge health surveillance. It has a profound significance to the development of health surveillance and safety assessment.

\section{Acknowledge}

This work was supported by National Natural Science Foundation (51208538, 51278512), Science and Technology Innovation Talents of Chongqing (CSTCKJCXLJRC17), cstc2015jcyjBX0014, cstc2016jcyja0022, the State Key Laboratory Breeding Base of Mountain Bridge and Tunnel Engineering(Chongqing Jiaotong University:CQSLBF-Y14-1)

\section{References}

[1] Yingxin Zhou. Study of Key Structure Safety Monitoring Technology of Mountain Roads [D]. Chongqing Jiaotong University, 2013.

[2] Bin Xue. Research and Application of Bridge Structural Degradation Evaluation Based on Vibration Detection [D]. Hebei University of Technology, 2008.

[3] Wenquan Deng. Design and Implementation of Data Acquisition System [D]. Taiyuan University of Technology, 2014.

[4] Shuo Jia. Design and Development of Structural Health Monitoring System Software for Sea Crossing Bridge [D]. Beijing Technology and Business University, 2008.

[5] Juanxia Li. Research on Bridge Health Monitoring System and Data Fusion Technology[D], Lanzhou University of Technology, 2008.

[6] Kang Xie. Research on Multi - license Plate Location Method of Intelligent Transportation System Based on CNN[D],Qingdao University,2011.

[7] Jianfan Lin, Chaowei Chen. Development of Safety Assessment and Management Program for Existing Bridges[A]. China Highway and Transportation Society, 2008. 